\title{
BIOÉTICA, TECNOCIENCIA Y PROCESO DE GLOBALIZACIÓN
}

\author{
BIOETHICS, TECHNOSCIENCE AND GLOBALIZATION PROCESS
}

\author{
GILBERTO CELY GALINDO \\ Pontificia Universidad Javeriana \\ gcely@javeriana.edu.co
}

RECIBIDO: $14 / 07 / 2016$

ACEPTADO: 29/07/2016

Resumen: La cultura dominante en el mundo contemporáneo está jalonada por el conocimiento científico y tecnológico, íntimamente unidos como tecnociencia. Esta cultura ha venido a llamarse "Sociedad del conocimiento", cuya visibilidad en los últimos tres siglos han sido las tres revoluciones industriales y estamos ad portas de iniciar una cuarta. Todas ellas con tendencia a la globalización. La Bioética nace para darles acompañamiento sapiencial.

Palabras claves: Bioética, tecnociencia, globalización.

\begin{abstract}
The dominant culture in the contemporary world is marked by scientific and technological knowledge, intimately united as technoscience. This culture has been called "knowledge society", whose visibility in the last three centuries have been the three industrial revolutions and we are $\mathrm{ad}$ portas (on the verge) of starting a fourth. All with globalization trend. Bioethics was created to give them wisdom support.
\end{abstract}

Key words: Bioethics, technoscience, globalization.

\section{Y nace la Bioética}

En 1927, el teólogo luterano alemán Fritz Jahr, introduce el neologismo BioEthik en un artículo que publica en el semanario Kosmos. Y en 1971, Van Rensselaer Potter, bioquímico norteamericano investigador en cáncer, de la Universidad de Wisconsin, desconociendo las ideas de Jahr, da a luz su famoso texto Bioethics, bridge to the future, seguido de Global Bioethics. Building on the Leopold Legacy, ocho años después.

Potter escribió el libro Bioética global, con el propósito de recuperar la herencia intelectual ecológica del ingeniero forestal Aldo Leopold y reivindicar para la ética contemporánea un conocimiento holístico sapiencial, interdisciplinario, de profunda raigambre ecológica, orientador del quehacer de los científicos e iluminador de la opinión pública, confiando en que la Bioética se convirtiese en un movimiento social o una actitud cívica.

Recordemos que uno de los errores crasos de la Modernidad fue haber enemistado y distanciado las ciencias de las humanidades, trayendo con esto una 
profunda crisis ética de la sociedad contemporánea. Más aún, la Modernidad supervaloró el desarrollo de las ciencias y minusvaloró los saberes humanísticos y filosóficos, en los cuales se inscribe la ética, e introdujo con Bacon principalmente, una actitud arrogante del quehacer científico. Al respecto dice Josetxo Beriain: "La Modernidad se sustenta sobre una infraestructura imaginaria, la expansión ilimitada del dominio racional que funge como racionalización de la 'voluntad de dominio'. Esta penetra y tiende a informar la totalidad de la vida social (por ejemplo, el Estado, los Ejércitos, la educación, etc.), a través de la revolución perpetua de la producción, del comercio, de las finanzas y del consumo".

Esta arrogancia es una de las enfermedades que afectan el desarrollo del conocimiento y requiere de una fuerte dosis de humildad, como lo expresa Potter en su libro Bioethics, Bridge to the Future, donde hace un llamado a la conciliación de las ciencias con las humanidades, ${ }^{2}$ con el propósito de lograr una visión interdisciplinaria ${ }^{3}$ e integradora del conocimiento. Dice Potter: "Concluyendo, lo que les pido es que piensen la Bioética como una nueva ética científica que combina la humildad, responsabilidad y competencia, en una perspectiva interdisciplinar e intercultural y que potencializa el sentido de humanidad". 4

A partir de Van Rensselaer Potter, la palabra Bioética comienza a darle velozmente la vuelta entera a nuestra casa terrenal. Está penetrando todos los resquicios de las ciencias biomédicas, de los procesos investigativos de las tecnociencias ${ }^{5}$ y sus aplicaciones prácticas, se hace imprescindible en las

\footnotetext{
${ }^{1}$ BERIAIN, Josetxo, (1996), en el Prólogo del libro Las consecuencias perversas de la modernidad, del cual Beriain es compilador, Anthropos, Barcelona, p. 12.

2 "Hay dos culturas - ciencias y humanidades- que parecen incapaces de hablarse una a la otra y si ésta es parte de la razón de que el futuro de la humanidad sea incierto, entonces posiblemente podríamos construir un 'puente hacia el futuro' construyendo la disciplina de la Bioética como un puente entre las dos culturas. Los valores éticos no pueden ser separados de los hechos biológicos". POTTER, Van Rensselaer, (1971) Bioethics, bridge to the future, Englewood Cliffs, N.Y., Vol. 1. XIX, Prentice Hall, p. 9.

3 "La Bioética debería llegar a ser un nuevo tipo de ciencia de la vida, esencialmente interdisciplinar y preocupada por la supervivencia de la especie humana, capaz de integrar la biología humana (ampliamente entendida) la competencia humana en crear y acompañar los valores humanos, los problemas del medio ambiente y de los otros seres vivos que intervienen en la preservación y mejoría de la propia calidad de vida de los seres humanos", al mismo tiempo "que un puente entre la cultura científico-técnica y la cultura humanística". POTTER, Van Rensselaer, (1971) Bioethics, bridge to the future, Englewood Cliffs, N.Y., Vol. 1, XIX, Prentice Hall, p. 8.

${ }^{4}$ Vídeo enviado por Potter al IV Congreso Mundial de Bioética, Tokio, noviembre 4-7, de 1998.

${ }^{5}$ Remito al lector a lo mejor que se ha escrito sobre el concepto de "tecnociencias" y al por qué la Bioética se configura como la ética propia del mundo tecnocientífico. OTTOIS, Gilbert (1991) El paradigma bioético. Una ética para las tecnociencias, Anthropos, Barcelona.
} 
reflexiones de la ecología, cuestiona con criterios de justicia y equidad los intrincados juegos económicos que orientan la dinámica del proceso de globalización, se sienta a la mesa de toma de decisiones macro de la biopolítica, y se ocupa de un larguísimo etcétera de temas que afectan el mundo de la vida biológica y cultural.

La Bioética se ocupa de cuidar la vida, su calidad y su sentido. Todo tipo de vida. Por supuesto la vida humana y los soportes abióticos. Porque todo está relacionado con todo en procesos de interacción e interdependencia. La vida nos pertenece como don, en cuanto su anterioridad es fundamento de nuestra existencia y rebasa toda posibilidad de apropiación exclusiva en la individualidad de cada uno de nosotros. La vida nos vincula radicalmente con todo lo biótico y abiótico y accede, en el ser humano, al estadio evolutivo de la conciencia intencional que permite la posibilidad de pensarse a sí misma y de asumir la elaboración de su devenir, confiando su suerte a las decisiones responsables y libre-relacionadas de cada hombre y de la colectividad humana.

Si la vida de nuestra casa terrenal está en alto riesgo por la acción humana tecnocientífica que magnifica sus impactos negativos en todo el planeta, la Bioética responde al reclamo que hace la humanidad de establecer mejores relaciones entre naturaleza y cultura, a favor de ambas. Las tecnociencias son productos culturales, es decir, construidos por el animal cultural, el humano, quien a la vez es construido por su producto. Dicho de otra manera, toda acción humana es modificadora tanto del mundo exterior, el hábitat, como del interior, la psique, y comporta responsabilidad moral con las presentes y futuras generaciones.

De la interacción del hombre con el hombre y con el hábitat natural y construido, surge un sistema complejo de símbolos que dan sentido a la vida personal, a la historia y al mundo. Una cosmovisión. Eso es la cultura. La forma práctica y simbólica como un pueblo vive, se organiza, se entiende a sí mismo y celebra su existencia. La cultura es la red aglutinante de expresiones humanas que dan coherencia, identidad y pertenencia al individuo con un grupo social, con base territorial. Es la manera como las personas adquieren una mentalidad y unas actitudes que guían su vida, su acción y sus relaciones. En el fondo de la cultura están los valores que dan soporte ético y estético al discernimiento y al buen gusto de vivir, que generan el juicio de lo justo y lo injusto, de lo bueno y lo malo, lo necesario y lo conveniente, lo honesto y lo deshonesto, lo aceptable y lo rechazable...

La Bioética se ha ido posicionando, entonces, como el nuevo nombre de la 
ética contemporánea que se las arregla con problemas complejos de la cultura tecnocientífica, en defensa de todo tipo de vida a favor de un éthos vital que tenga calidad y sentido. El prefijo griego Bios se convierte en el imperativo categórico para la calidad del éthos vital que deseamos vivir las presentes generaciones, pensando también en las futuras. El cuidado del medio ambiente es fundamental para la sustentabilidad de la vida en el planeta.

Toda cultura, todo pueblo, toda persona construye históricamente su éthos vital: su moral. Vale decir, su modo de morar, de habitar la casa terrenal y simultáneamente su casa interior: su conciencia, su espiritualidad, su religiosidad. Moradas estas del éthos vital con las que avanza o retrocede en el proceso de humanización, para dotarse de sentido existencial trascendente y aspirar a gozar de una vida con calidad para las actuales y futuras generaciones.

El éthos vital contemporáneo es un complejo constructo social, mediado por conquistas tecnocientíficas a merced de la dinámica económica dominante, con alto poder de fascinación del imaginario colectivo en irresistibles promesas de bienestar que masajean estratégicamente el mundo insaciable del deseo, del consumo sin límites, de la avaricia.

\section{La sociedad tecnocientífica y sus discursos}

El discurso de las ciencias económicas y administrativas de la globalización tiene a su favor una lógica que ilusiona y conquista voluntades porque articula sagazmente la democracia con la tecnociencia y la tecnocracia a favor de mejores condiciones de vida. De vida buena, saludable, longeva y placentera para todos los seres humanos y países que cooperen con la dinámica de globalización. Democracia y tecnociencia en abundancia. Ambas cogidas de la mano como matrimonio inseparable.

Con la democracia, como sistema sociopolítico, se promueven todos los anhelos de libertad que anidan en el corazón humano: autonomía, libertad de pensamiento y expresión religiosa, política y cultural, ejercicio de derechos de propiedad privada, de auto-organización, de libre asociación empresarial, de elección popular de gobernantes, de protección de la vida, la honra y los bienes de los ciudadanos, y un sinfín de condiciones políticas que garantizan las opciones preferenciales de las gentes.

Con la tecnociencia, vienen todas las promesas de progreso económico, de bienestar, de superación de toda precariedad y contingencia humanas, las condiciones de bienestar material, de estilo de vida confortable, de abundancia de bienes y servicios, el combate a muerte contra las enfermedades y la muerte 
gracias a los grandes avances en higiene, nutrición, farmacopea y cirugías. Además de los ingentes esfuerzos por superar la ignorancia y los tabúes que restan libertad al ser humano; de correr permanentemente las fronteras del conocimiento sobre el hombre y el cosmos y de apropiarse del mundo, de sí mismo y de la dirección de la historia. "Desde hace dos siglos, la industrialización la preparaba, pero sólo hace unos treinta años que la técnica se impone por doquier, lo modifica todo, se apropia de todas las actividades y formas sociales y se convierte en un verdadero medio para el hombre". ${ }^{6}$

Del estrecho abrazo entre ciencia y técnica modernas, y del inmenso poder que de allí surge para sus gestores, la idea de progreso continuo e ilimitado empuja el proceso de globalización, generando la premisa de que todo lo que se desee conseguir se logrará si se tiene fe en la tecnociencia, pues gracias a ella "siempre hay algo nuevo y mejor para encontrar". ${ }^{7}$ Este principio se sustenta en una visión teórica "de la naturaleza de las cosas y del conocimiento de éstas, según la cual éstas no ponen ningún límite al desarrollo y la invención, sino que más bien abren en cualquier punto a partir de ellas un nuevo acceso a lo que queda por conocer y por hacer". 8

\section{¿Qué estamos globalizando?}

Así que, democracia y tecnociencia unidas conforman un poderoso discurso que vocifera la economía en favor de sus propuestas de globalización. Discurso además ideológico por su capacidad de fascinar, de encantar, de hacer promesas mágicas, de ilusionar con visiones paradisíacas de una vida mejor aparentemente para todos, pero en la realidad para unos pocos privilegiados que concentran capitales. Y muy bueno también para algunos países expertos en crear las condiciones de la "Cuarta revolución industrial" que estamos comenzando. Se trataría esta última de la revolución pacífica más acelerada, audaz, imparable, global, incontrolable y de impactos desestabilizadores de la arquitectura sociocultural vigente por su inmensa capacidad de modificar globalmente los sistemas simbólicos de la moral, la ética y la estética de todas las culturas.

Sucedió que la "Tercera revolución industrial” (¡inconclusa todavía...!) gran ícono de la Modernidad, no ha cumplido todavía con sus promesas de bienestar

\footnotetext{
${ }^{6}$ ELLUL, Jacques, Ce que je crois, Grasset et Fasquelle, Paris, 1987, p. 179. Por "medio" Ellul entiende "aquello que permite al hombre vivir, y que lo pone en peligro, aquello que le es inmediato, y que mediatiza todo lo demás".

${ }_{7}^{7}$ JONAS, H., (1996) Técnica, medicina y ética, Paidós, Barcelona, p. 21.

${ }^{8}$ JONAS, H., Ibidem, p. 21.
} 
para todos los seres humanos. Pero sí ha despilfarrado ingentes riquezas en armamentismo altamente destructor, en guerras de terrible violencia genocida, sumas gigantescas en exploración espacial, produjo el calentamiento global y arruinó el medio ambiente con la falsa conciencia de que el planeta dispone de recursos naturales inagotables. Además, el siglo XXI lo comenzamos dejando deudas para que las paguen las próximas tres generaciones de jóvenes. Todo esto y mucho más ocasionó la aparición cultural de la Posmodernidad, ${ }^{9}$ como desencanto ante el incumplimiento de los metarrelatos ideológicos de la Modernidad.

En esta línea de pensamiento, la economista Karen Gloy afirma que la economía dominante del proceso de globalización crea una ideología inspirada en la corriente de la Ilustración, un nuevo dogma de fe que Gloy llama "monomitos". ${ }^{10}$ Es decir, mitos monotemáticos en torno a una especie de mesianismo salvador universal, que pueden ser de tres clases. Un monomito de estilo de vida ilusoriamente deseable para todas las gentes del globo, que no es otro que uniformar con el American way of life. Otro monomito que confronta, sin posibilidad de otra opción, vivir de una forma moderna, en libertad; o vivir sometido a integrismos conservadores que comportan formas precarias de vida y retrasan el desarrollo histórico de la humanidad. Un tercer monomito globalizador habla de multiculturalismo como ideal de vida, bajo el supuesto de que todos los valores y culturas valen lo mismo, merecen el mismo respeto, lo pequeño es grandioso y lo deseable es ser ecléctico y multicultural.

Este ideal de globalización trae a pérdida las identidades de los grupos y pueblos, diluye las jerarquías de valores morales que han aportado fortaleza a las comunidades y etnias para superar históricamente sus vicisitudes. Hace creer que la expectativa de vida buena consiste en borrar las fronteras de las creencias personales que nos identifican y diversifican, para asumir sincréticamente los

\footnotetext{
9 "La posmodernidad, en un primer momento, se revela como un desencanto frente a la modernidad, que representaba el mito del progreso a partir de la razón. Frente a los excesos de la racionalidad se apuesta la sensibilidad y a la afectividad, frente a proyectos totalizantes, se prefiere dar valor a la diversidad, frente a la preocupación por el progreso y por el futuro, se da más atención al presente en toda su densidad; y cuando se apuesta por la libertad se radicaliza, desemboca en un individualismo que reduce a la sociedad a un conglomerado de individuos preocupados solamente con su intereses particulares y menos sensibles a los intereses generales de la colectividad: Esta es una característica muy típica del mundo de hoy". Adroaldo Palaoro, S.J., en Los Ejercicios Espirituales en el Contexto Posmoderno. Una espiritualidad Encarnada e Integradora, pg. 1. Traducción del Portugués - Pedro Nel Ortiz L., S. J. http://www.cpalsj.org/wp-content/uploads/2013/04/201302Itaici-Los-EE-en-elcontexto-Posmoderno.pdf Consultado 19-05-2016.

${ }^{10}$ GLOY, Karen, Globalização - mais como?, cadernos IHU idéias, UNISINOS, São Leopoldo, Brasil, ano 4-Nº 59, 2006.
} 
valores y formas de vivir de los pueblos llamados desarrollados, perdiendo toda raigambre histórica y locativa con un grupo cultural de pertenencia.

Posiblemente esta especie de "cultura universal, globalizada y homogeneizante" traiga más bien desarraigo afectivo, liviandad del ser, vacío existencial, anonimato, anomía y una forma plana de vivir. "Lo afectivo parece hoy más frágil, más vulnerable, más efímero y más ausente. Hay mucha sed insatisfecha, mucha incomunicación, bastante soledad no buscada y bastante fracaso en las relaciones, algo que deja a las personas heridas y abatidas". ${ }^{11}$

El sistema económico global tecnocientífico, cimentado en la razón instrumental, ${ }^{12}$ tiende a crear su propia ética técnica. Parte del axioma de que todo lo que sea técnicamente posible es éticamente deseable. Por otro lado, la ética técnica no se suma a las éticas propias de las culturas no técnicas, sino que las suplanta, enrareciendo sus jerarquías de valores que han dado soporte ancestral al tejido social.

Para Jacques Ellul, la ética técnica "no produce una simple adhesión de valores nuevos a valores antiguos (...) Estas civilizaciones antiguas se hunden en contacto con la técnica (...) pues disocia las formas sociológicas, destruye los marcos morales, hace explotar los tabúes sociales o religiosos, desacraliza a los hombres y las cosas y reduce el cuerpo social a una colección de individuos". ${ }^{13}$

Podríamos decir que el neoliberalismo económico globalizado no sabe del gran valor bioético de la solidaridad. O sí sabe, pero no lo aplica en serio porque sobrepasa sus temores, ya que la concentración del capital ${ }^{14}$ es su estrategia de supervivencia. Repartir justamente y con equidad la riqueza sería un acto suicida para el sistema. Usa la palabra solidaridad en sus discursos para acallar

\footnotetext{
${ }^{11}$ RODRÍGUEZ OLAIZOLA, José María, S.J. (2011) Hoy es ahora (gente sólida para tiempos líquidos), Sal Terrae, España.

${ }^{12}$ Max Horkheimer (1895 - 1973), sociólogo y filósofo alemán, echó a andar en 1947 el concepto de "Razón instrumental", como crítica filosófica a la creciente cultura tecnocientífica que privilegia todo lo útil, lo práctico, lo económicamente productivo, con sus conceptos de eficiencia y eficacia en orden a la productividad. Lo valioso es lo que rinde resultados evaluables y las cosas valen en función de este criterio, con el riesgo de presumir que el ser humano queda subsumido en esta misma percepción que objetiviza y convierte en fines los medios. También el Theodor Adorno (1903 1969) y otros filósofos de la Escuela de Frankfurt adhieren a la propuesta de Horkheimer.

${ }^{13}$ ELLUL, Jacques, (1990) La technique ou l'enjeu du siècle, Economica, Paris, p. 115.

14 "Mano a mano con la centralización del capital (...) se desarrolla, en una escala cada vez más extensa, (...) la implicación de todas las personas en la red del mercado mundial y con ello el carácter internacional del régimen capitalista. Junto con el constante aumento del número de magnates del capital que usurpan y monopolizan todas las ventajas de este proceso de transformación, aumenta la miseria, la opresión, la esclavitud, la degradación y la explotación." CAPRA, Fritjof, (1998) El punto crucial. Ciencia, sociedad y cultura naciente, Editorial Troquel S.A., Buenos Aires, en el capítulo "Callejón sin salida de la economía", pp. 233-234.
} 
conciencias, pero en realidad lo que ofrece son "falaces ayudas económicas" o limosnas paliativas que no remedian la pobreza, la indigencia y la dependencia de las mayorías respecto de las minorías ricas.

El capitalismo le tiene pánico a la solidaridad porque considera que se convertiría en una decisión suicida, en una especie de muerte entrópica de la dínamis económica, es decir, de un equilibrio de fuerzas que paralizaría el mercado.

La economía liberal se fundamenta en la lógica de acumulación y retención de capital, es decir, en riqueza para hacer más riqueza de manera especulativa hasta bordear los límites del riesgo. Para ello recurre a las teorías de la competitividad, de la eficiencia, de la eficacia, de la innovación y de la creatividad para aumentar la productividad y el consumo. Su tendencia es a conformar oligopolios. Y, falazmente, después repartir beneficios, sin abandonar la concentración de capital. ¿Es esto posible, sin contradecir el sistema? ${ }^{15}$

En la práctica, la supervivencia del capital no permite repartir beneficios ni siquiera con los que son accionistas, porque si reparte todo se queda con nada y pierde su músculo financiero o dinámica de inversión, sin la cual no se reproduce la riqueza y se entra en lo que llamaríamos "muerte entrópica" del modelo económico. Por demás, si se solidariza con los pobres, que son la mayoría del mundo, haciéndolos socios, haría su peor negocio, pues ellos no cuentan con ahorros para invertir, solamente consumen pobremente. Así que los pobres no son sus aliados, sino pequeños clientes, consumidores de baratijas y empleados de oportunidad.

Los pobres son buenos como mano de obra abundante y barata, a quienes cada vez más los reemplazan con máquinas industriales, robots y tecnología informática. Las máquinas son más eficientes, no protestan por malos salarios, no hacen huelgas porque no conforman sindicatos y son fieles a sus amos. La "Cuarta revolución industrial" fijará sus esperanzas en las máquinas, la robotización, en la información veloz, la nanotecnología, la inteligencia artificial y el capital humano muy especializado. ¿Qué pasará entonces con la inmensa mayoría de gente que perderá su empleo por culpa de todo lo anterior? Las empresas serán cada vez menos en cantidad y en número de personas laborando. Los países avanzados tendrán menos jóvenes y más ancianos. Las migraciones

\footnotetext{
15 "Los defensores de la globalización neoliberal sostenían que el crecimiento le llegaría a todo el mundo. En cambio, se concentró cada vez más en un número creciente de manos. En 2010, 388 personas concentraban la riqueza de 3.600 millones de personas. En 2014, ese número se redujo a 80 personas, y en 2015, a 62.” Roberto Savio (periodista argentino), ¿Cómo llegamos a este caos?, en http://www.cpalsocial.org/1320.html

Consultado 8 de agosto 2016.
} 
internas y externas aumentarán y los conflictos sociales serán mayores.

Cuando los empleados elevan sus justos reclamos, y/o las garantías de seguridad no son fiables para la inversión, y/o las leyes nacionales son estrictas y no logran burlarlas, o ya se han extinguido los recursos naturales del lugar, el capital huye del país y se ubica en territorios de menores exigencias y mejores condiciones para sus objetivos.

Cada vez más el capital es cosmopolita, multinacional, transnacional, desterritorializado, se traslada a su antojo a cualquier parte del mundo por medios electrónicos y su patria son los paraísos fiscales donde pueda rendir más y tributar menos. A la postre, el capital solamente es solidario con sus propietarios. No lo es con los seres humanos que desgastan su fuerza laboral en producirlo. Y menos con el medio ambiente que termina vejado sin reparación alguna.

En resumen, el sistema económico es mundial. Vive de la especulación, la maximización del lucro, la codicia y el miedo, la rentabilidad financiera, la falsa ilusión del crecimiento ilimitado, del consumismo y de la idolatría del mercado. Desconoce que los recursos naturales son finitos, que el deterioro del medio ambiente con el cambio climático está llegando a la etapa del no retorno en el calentamiento global y que todo lo anterior atenta contra la dignidad humana sin distingos de pobres y ricos. La Bioética, pues, alza su voz contra estas prácticas económicas globales que causan severos e irreversibles daños ecológicos y humanos. El sistema es perverso porque destruye la jerarquía de valores que dan soporte moral a los individuos y al todo social.

\section{Globalización que debilita el espesor moral de la sociedad}

El hombre contemporáneo de la aldea global queda a merced del individualismo creciente para defenderse como pueda de la anomía social, en reacción a la sociedad tecnológica que se edifica sobre la negación de la diferencia, por su lógica de eficiencia y productividad competitiva. Esto sucede mientras la fuerza organizacional de la tecnológica sociedad global promueve procesos homogeneizantes de las costumbres, reparte migajas de identidades personales con pequeños modelos fragmentados que sustituyen matrices culturales $\mathrm{y}$ religiosas, las cuales ofrecían otrora alta coherencia afectiva y moral. El proceso de globalización no suprime en realidad las identidades, sino que las dispersa, deshilachando y debilitando la unidad interior de los seres humanos.

La publicidad comercial y política disemina la ideología, convenciendo a la gente que trabaje y se esfuerce diariamente para acceder a la felicidad que se 
logra adquiriendo cuantas ofertas le hace el mercado. ¿Y cuál es la oferta del mercado? Libertad, autonomía y bienestar representadas en multiplicidad de objetos y oportunidades de confort que brillan como oro, pero son falso oropel: electrodomésticos, aparatos de música y video, celulares sofisticados, computadores, vehículos, ropa de marca, viajes turísticos, deportes, estética, salud, comidas suculentas y una lista enorme de satisfactores de gustos servidos a la carta.

Detrás de todo lo anterior hay una lucha cotidiana de trabajo excesivo para conseguir dinero y luego gastarlo en consumismo, hedonismo, narcisismo, permisividad sexual, drogadicción, subjetivismo y relativismo moral. Este es el oropel que lleva a la fragmentación del sujeto, a la pérdida de sentido y significado de la vida, a la banalidad. Todo lo anterior socava la autoestima individual y le resta espesor moral a la sociedad.

Este sinsabor existencial ocasiona que aparezca en el mercado de hoy en día un vasto bazar de ofertas de sentido para condimentar la vida sosa, recuperar la autoestima y tratar de apuntalar el andamiaje social. El menú es grande y variado para todos los gustos y capacidades económicas. Están los consultorios de psicólogos, psiquiatras y consejeros afectivos y espirituales. Las librerías abundan en literatura de autoayuda, Nueva Era, medicina naturalista, esencias florales, alimentación vegetariana, recreación al aire libre, ecologismo barato y novelas de espiritismo. La prensa publica diariamente el horóscopo, las predicciones astrológicas, el zodíaco y cuanto estimule la esperanza de superar frustraciones y mejorar la suerte. Proliferan las loterías y rifas legales (y de las otras...!) para mantener la ilusión colectiva de enriquecimiento mágico y caído del cielo. También abundan los espectáculos musicales, teatrales, deportivos, cines, t.v., internet, exposiciones culturales para distraerse y gastar el escaso tiempo libre; más las historietas pasionales para lectores ansiosos que buscan soluciones fáciles a sus problemas humanos difíciles. No faltan (en pleno siglo XXI de la Sociedad del Conocimiento tecnocientífico altamente ilustrado) los hechiceros, brujas, chamanes y adivinos de la suerte con sus amuletos, talismanes, bebedizos, baños, rezos y predicaciones milagrosas con que engañan y explotan a las gentes ingenuas y sugestionables.

Por otra parte, están de vuelta la religión y la espiritualidad de las grandes iglesias históricas; como también proliferan las nacientes sectas religiosas pentecostales brasileñas que venden sus franquicias a elocuentes pastores criollos que van tras los diezmos y limosnas de los conversos. Están de vuelta los integrismos, las luchas nacionalistas, los populismos, el terrorismo, los grupos guerrilleros que reivindican el poder para el pueblo oprimido y tantas otras agrupaciones que atraen a insatisfechos sociales. También hay un creciente 
clamor por una ética mundial que cuide preferentemente del mundo de la vida bilógica-cultural. Este es el gran reclamo de la Bioética.

La universalidad de la fe en las capacidades cognitivas humanas, y sólo en ellas, es un credo de la razón ilustrada de la Modernidad, que se expresa como luz iluminadora universal de las conciencias, en busca del dominio absoluto de la naturaleza para liberarse de las condiciones que ésta interpone a los seres humanos. El hombre es capaz por sí mismo de resolver los problemas de su ser en el mundo, en la medida en que vaya apropiándose del mundo, incluyendo el de su propia interioridad. Éste es el dogma principal del antropocentrismo moderno que galopa a horcajadas en el brioso corcel de la globalización y que el Papa Francisco critica como "tecnocracia", en la Encíclica Laudato Si. ${ }^{16}$

A la postre, la vida del adulto contemporáneo se recarga de inmanencias fatigantes que dejan poco espacio para cultivar experiencias de trascendencia, de autocomprensión y de proyectos liberadores de orden existencial. Estos déficits deterioran la sociedad sólida y relativizan los compromisos de largo aliento como la fidelidad matrimonial, las relaciones filiales, las auto-obligaciones morales de votos religiosos perpetuos, las adhesiones a partidos políticos, la identidad y pertenencia institucionales, el sentido de patria y todos aquellos valores culturales que exigen esfuerzo constante.

¿Y qué pasa, mientras tanto, con los jóvenes? Especialmente con aquellos que han padecido una crianza sin afecto y malos tratos. Aquellos que son huérfanos con padres vivos. Quienes han recibido muchos juguetes electrónicos y concesiones a sus caprichos a cambio de tolerar conductas incorrectas de sus padres. Quienes han aprendido más cosas acerca de los misterios de la vida en los corrillos de compañeros en las calles que en sus hogares y escuelas. En fin, con todas aquellas chicas y chicos fastidiados con el tipo de sociedad que los adultos les estamos dejando como herencia. Sociedad líquida. ${ }^{17}$ Los jóvenes son críticos implacables de nuestros modos de vida y de las onerosas cargas de

\footnotetext{
${ }^{16}$ A continuación, presento algunos conceptos del Papa Francisco, en su Encíclica Laudato Si, sobre la "tecnocracia". La tecnociencia se ha vuelto tecnocracia, una verdadera dictadura con su lógica férrea de dominio sobre todo y sobre todos (n.108). El gran límite de la tecnocracia está en el hecho de «fragmentar los saberes y perder el sentido de totalidad» (n.110). La mayor desviación producida por la tecnocracia es el antropocentrismo. Este supone ilusoriamente que las cosas solo tienen valor en la medida en que se ordenan al uso humano, olvidando que su existencia vale por sí misma (n.33).

${ }^{17}$ El sociólogo polaco Zygmunt Bauman describió estos comportamientos y acuñó el apelativo de "Modernidad líquida" para caracterizar las tendencias sociales contemporáneas que parten del primer mundo y rápidamente se riegan por el resto, destruyendo los valores sólidos de la estructura humana. Tendencias que no son exclusivas de los jóvenes. Muchos adultos que deberían pertenecer a la "Sociedad sólida" de las gentes mayores son los gestores ocultos del relativismo moral que van haciendo líquidas y gaseosas las costumbres. En esto consiste su estrategia económica para llenar sus bolsillos.
} 
neurosis que ponemos sobre sus espaldas para que sobrelleven penosamente nuestro futuro incierto.

\section{¿La Ilustración quedó inconclusa y todavía está en marcha...?}

Finalmente, y para que no quede flotando una atmósfera negativa y pesimista sobre la Modernidad tardía que vivimos actualmente, con sus tres Revoluciones industriales y la Cuarta incipiente, hagamos un recuento sumario de los beneficios que nos deja en herencia. Vale aclarar que no podemos disociar la Modernidad de la Ilustración, ni estas dos de las tecnociencias que han venido comprometiendo su rostro con los procesos industriales que globalizan todo este conjunto de logros históricos. El ensayo de Michel Foucault (1984, "Qu'est-ce que les Lumières?") sobre el concepto Kantiano acerca de la Ilustración, transmite la convicción de que es una etapa de la humanidad que sigue avanzando.

La Ilustración fue y seguirá siendo un estallido universal imparable y permanente de autoconciencia de lo que nos hace humanos adultos: la inteligencia que razona con propiedad sobre la naturaleza del hombre y de las cosas, el abandono del pensamiento supersticioso e ingenuo, la ruptura de todas las esclavitudes que impiden el ejercicio de la voluntad libre, la autonomía, la dignidad inalienable de cada persona y de los pueblos, la democracia política, los Derechos humanos y la educación de calidad para el logro de todo lo anterior. "La ilustración es la salida del hombre de su autoculpable minoría de edad...", decía Emmanuel Kant. Ninguno de estos propósitos del Siglo de las Luces se ha culminado a cabalidad. Luego la tarea sigue pendiente y en marcha. No podemos simplemente pasar la página. Pienso que la Bioética tiene vocación de puentear la contemporaneidad tecnocientífica universalizada con el Humanismo científico de la Ilustración, a favor de una visión integral del ser humano.

La Ilustración no fue un simple puñado de acontecimientos episódicos de finales del siglo XVII y todo el XVIII, que siguió al Renacimiento y culminó con la Revolución francesa. ¡No! Sino una revolución humanística dinámica que sigue su marcha sin fin, transformando mentes, corazones y estructuras sociopolíticas como una luz resplandeciente que destruye las ciegas tinieblas de la ignorancia para construir mejores futuros de libertad, justicia, desarrollo socioeconómico y vida buena. A los grandes pensadores y hombres de ciencia que encendieron la antorcha de la Ilustración en Europa ${ }^{18}$ tendríamos que agregar una

${ }^{18}$ Descartes, Locke, Bacon, Bayle, Galileo, Grocio, Hobbes, Leibniz, Newton, Spinoza, Kant..., y 
lista muy larga de personajes a nivel mundial que han continuado esta carrera imparable de relevos en las ciencias y humanidades, en los últimos dos siglos largos. "Sapere aude": ten valentía para servirte de tu propio entendimiento. Esta fue la consigna del Siglo de las Luces. Consigna que sigue vigente.

A partir de los años treinta del siglo pasado, las ciencias físicas y biológicas emprendieron su veloz carrera con todo tipo de aplicaciones tecnológicas hasta convertirse en punta de lanza de una nueva sociedad y benefactoras de esta: la Sociedad del Conocimiento con sus revoluciones industriales y su liderazgo en la gestión del modo de pensar, sentir y actuar de las gentes actuales, guiadas por el pragmatismo fruitivo de lo útil y lo factible, el cambio permanente y acelerado, y el gozo sin mesura de los bienes de la tierra re-creados industrialmente.

Por su utilidad práctica y capacidad seductora, las tecnociencias se han ido apoderando del imaginario colectivo que estrecha la brecha entre el ser y el deber-ser, de lo real y lo posible, lo razonable, para decirnos lo que es bueno y lo que es malo, lo correcto e incorrecto, y lo que es o no funcional para satisfacer las necesidades humanas. Es así como las tecnociencias avanzan en ganar escaños propios en los juicios de valor moral y del pensamiento ético, tareas que tradicionalmente han sido de la filosofía y la teología. A esto se refiere Jacques Ellul, con su concepto crítico de la ética técnica.

La Ilustración abrió y no ha cerrado el espacio del conocimiento científico y tecnológico a favor de resolver nuestras necesidades en salud, vivienda, alimentación, vestuario, comunicación social, movilidad y transporte, trabajo digno, descanso, recreación, y un larguísimo etcétera de novedades tecnocientíficas que nos sacan de la pobreza, alivian las fatigas y proporcionan calidad de vida, aparentemente para todas las gentes del planeta. Pero estamos lejos de que esto último acontezca.

Toda esta maravillosa gesta de la inteligencia humana se ha centrado en la confianza absoluta en la razón, como medio convertido en fin del modo de conocer ilustrado para intervenir lo conocido. Esta absolutez ha puesto a la razón a caminar en la sinrazón. ${ }^{19}$ El método científico marcó la diferencia entre las

agreguemos algunos de los científicos que desarrollaron la física cuántica en la construcción de la bomba atómica: Carl Friedrich von Weizsäcker, Otto Hahn, Enrico Fermi, Robert Oppenheimer, Niels Bohr, Albert Einstein..., entre otros.

${ }^{19}$ Tenemos muchísimos ejemplos de episodios históricos recientes de sinrazón. Citemos algunos: el armamentismo y las dos guerras mundiales, las bombas atómicas de Hiroshima y Nagasaki, los campos de concentración nazi, los gulags de la Unión Soviética, el calentamiento global y la destrucción ambiental como consecuencias de nuestro estilo de vida industrializado, el consumismo voraz, las relaciones injustas de los países del Norte con los del Sur, el terrorismo, la instrumentalización del ser humano en la investigación científica, los fanatismos políticos y religiosos, etcétera-etcétera. 
ciencias positivo-analítico-experimentales y las ciencias sociales y humanas, llamadas también histórico-hermenéuticas o humanidades.

El método positivista y los sorprendentes resultados prácticos de su aplicación trajo un "endiosamiento" de las primeras y una especie de capitis diminutio de las segundas, con severas dificultades de diálogo e interacción entre ellas, además de cierto antagonismo con inculpaciones mutuas. Para resolver este conflicto viene la Bioética con su propuesta interdisciplinaria y transdisciplinaria, buscando aunar esfuerzos dialógicos para encontrar soluciones sapienciales a los problemas complejos de la Sociedad del Conocimiento.

Se espera, entonces, que la Bioética lidere un Humanismo científico que aporte luz para el discernimiento y toma de decisiones individuales y colectivas ante tres encrucijadas dilemáticas que nos acosan hoy: el cuidado de la vida, su calidad y el sentido existencial. 